\title{
Effects of Interactions and Temperature in Disordered Ultra-Cold Bose Gases
}

\author{
Clemens Adolphs',2, Joseph Towers', Marie Piraud1,3, Konstantine V. Krutitsky ${ }^{4}$, \\ David A. W. Hutchinson 1,5 \\ ${ }^{1}$ The Jack Dodd Centre for Quantum Technology, Department of Physics, University of Otago, Dunedin, \\ New Zealand \\ ${ }^{2}$ Department of Physics and Astronomy, University of British Columbia, Vancouver, Canada \\ ${ }^{3}$ Laboratoire Charles Fabry, Institut d'Optique, Centre National de la Recherche Scientifique, Université Paris \\ Sud, Palaiseau, France \\ ${ }^{4}$ Fachbereich Physik der Universität Duisburg-Essen, Campus Duisburg, Duisburg, Germany \\ ${ }^{5}$ Centre for Quantum Technologies, National University of Singapore, Singapore \\ Email: j.towers.nz@gmail.com
}

Received 25 November 2013; revised 23 December 2013; accepted 24 January 2014

Copyright @ 2014 by authors and Scientific Research Publishing Inc.

This work is licensed under the Creative Commons Attribution International License (CC BY).

http://creativecommons.org/licenses/by/4.0/

(c) (i) Open Access

\begin{abstract}
We simulate ultra-cold interacting bosons in quasi-one-dimensional, incommensurate optical lattices. In the tight-binding limit, these lattices have pseudo-random on-site energies and thus can potentially lead to Anderson localization. We use the Hartree-Fock-Bogoliubov formalism in the Bose-Hubbard model to explore the parameter regimes that lead to exponential localization of the ground state in a 3-colour optical lattice and investigate the role of repulsive interactions, harmonic confinement and finite temperature.
\end{abstract}

\section{Keywords}

\section{Ultra-Cold Atoms, Anderson Localization, Disorder, Optical Lattice}

\section{Introduction}

Cold atoms in optical lattices provide a great tool to study many theoretical models from solid-state physics as they allow for unique control of normally unmodifiable parameters [1]-[6]. Interactions can be tuned by a Feshbach resonance [7] or spatial confinement [8]-[10]. Disorder with known statistical properties can be introduced in a controlled manner which is usually done by purely optical means using either a laser speckle potential [11][13] or a secondary optical lattice incommensurate with the primary one [14]-[22]. It can be also formed by lo- 
calized impurity atoms [23].

Incommensurate lattices belong to a special class of potentials. Being completely deterministic, they can lead to the phenomena generic for random potentials. One of the famous examples is Anderson localization which can be understood as the effect of destructive interference of randomly scattered waves [24]. Several experimental groups have studied localization effects in ultra-cold Bose gases in incommensurate optical lattices [14][22]. In the ideal Bose gas, they are determined by the properties of the eigenstates of the single-particle Hamiltonian which were theoretically studied in Refs. [25]-[33]. Weakly interacting regime was investigated using the Gross-Pitaevskii equation in discrete [34] [35] and continuum models [19] [36] [37] as well as Bogoliubov theory [38]. The strongly correlated regime which may lead to quantum phase transitions was studied within the Gutzwiller mean-field approximation [39]. Exact numerical results for one-dimensional systems were obtained by quantum Monte Carlo [40], density matrix renormalization group [41]-[44], as well as by Bose-Fermi mapping in the limit of infinitely large interactions [40] [45] [46] [47]. Recently, the bichromatic model was numerically investigated in the presence of a synthetic gauge field, providing contrast to the two interference phenomena [48]. All previous theoretical studies mentioned above were performed at zero temperature. Only recently were finite-temperature properties of the Bose gas in a bichromatic optical lattice considered in the limit of vanishing tunneling or vanishing interaction [49].

The aim of the present work is to study weakly interacting Bose gas in an incommensurate optical lattice at finite temperature. We extend mean-field treatments [50] and their finite temperature generalizations [51]-[53], which have been extensively developed for the dilute Bose gas, including their application to the optical lattice [38] [54] [55], to include disorder.

\section{The System}

The setup we use for our simulations is taken from Ref. [16]. We consider an ultracold atomic sample in an elongated magnetic trap with longitudinal and transverse frequencies $\omega_{t}$ and $\omega_{\perp}$, respectively. If all energy scales do not exceed $\hbar \omega_{\perp}$, atoms occupy only the ground-state mode of the transverse confining potential and the system becomes effectively one-dimensional. The inter-particle interaction in the longitudinal direction can be well approximated by an effective two-body potential [8]

$$
V_{\text {int }}\left(x-x^{\prime}\right)=\frac{4 \hbar^{2} a_{s}}{m a_{\perp}^{2}}\left(1-1.4603 \frac{a}{a_{\perp}}\right)^{-1} \delta\left(x-x^{\prime}\right),
$$

where $a_{s}$ is the three-dimensional scattering length and $a_{\perp}=\sqrt{\frac{2 \hbar}{m \omega_{\perp}}}$ is the size of the ground state for the harmonic potential with the frequency $\omega_{\perp}$. Depending on the ratios $\omega_{\perp} / \omega_{t}, a_{s} / a_{\perp}$, and the density of atoms, the system can be in a quasi-one-dimensional regime which is well described by the mean-field theory, or in the Tonks-Girardeau regime where quantum fluctuations may be significant. Precise conditions for the Tonks-Girardeau regime were formulated in Ref. [9]. We note that in the experiment of Ref. [16], the quasi-one-dimensional regime was realized.

The potential of the primary optical lattice is

$$
V_{L}(x)=V_{0} \cos ^{2}\left(2 \pi x / \lambda_{L}\right),
$$

where $V_{0}$ is the lattice depth and $\lambda_{L}$ is the lattice wavelength. The disorder potential is of the form:

$$
V_{\text {dis }}(x)=V_{\text {dis }}^{0}\left[\cos ^{2}\left(2 \pi \alpha x / \lambda_{L}\right)+\cos ^{2}\left(2 \pi \beta x / \lambda_{L}\right)\right],
$$

where $\alpha=\lambda_{L} / \lambda_{\alpha}$ and $\beta=\lambda_{L} / \lambda_{\beta}$ are the ratios between the primary and secondary lattice wavelengths. For irrational values of $\alpha$ and $\beta$, the resulting potential is pseudo-random. In experiment, however, it is hard to make the wavelength ratios precisely irrational, so the potential exhibits an additional level of quasi-periodicity. If $\alpha$ and $\beta$ are ratios of large coprime integers, however, the period of the potential will be larger than the system size.

\subsection{The Bose-Hubbard Model}

The wave function is expanded in Wannier functions localized at the minima of the primary lattice potential in 
the lowest Bloch band. This tight-binding approximation leads to the Bose-Hubbard Hamiltonian for atoms in a one-dimensional optical lattice of $N$ sites,

$$
\hat{H}=\sum_{i=1}^{N} \hat{n}_{i} \epsilon_{i}-\sum_{i=1}^{N-1} J_{i, i+1}\left(\hat{a}_{i+1}^{\dagger} \hat{a}_{i}+\hat{a}_{i}^{\dagger} \hat{a}_{i+1}\right)+\frac{U}{2} \sum_{i=1}^{N} \hat{n}_{i}\left(\hat{n}_{i}-1\right),
$$

with tunneling energies $J_{i, i+1}$, on-site interaction parameter $U$, and on-site energy $\epsilon_{i}$, which contains both the trapping potential and the disorder potential. These parameters are determined by the lattice and disorder potential and are obtained by taking integrals over the Wannier functions [1] [41]. The resulting expressions, using a Gaussian approximation ${ }^{1}[56]$ for the Wannier functions, are:

$$
\begin{gathered}
U=4 \sqrt{2 \pi} \frac{\hbar^{2}}{m a_{\perp}^{2}} \frac{a_{s}}{\lambda_{L}}\left(1-1.4603 \frac{a_{s}}{a_{\perp}}\right)^{-1}\left(\frac{V_{0}}{E_{R}}\right)^{1 / 4}, \\
J=V_{0} \exp \left(-\frac{\pi^{2}}{4} \sqrt{\frac{V_{0}}{E_{R}}}\right)\left(\frac{\pi^{2}}{4}-1\right), \\
\epsilon_{i}=\epsilon_{\mathrm{dis}}^{0}\{\cos [\pi \alpha(2 i-1)]+\cos [\pi \beta(2 i-1)]\}+\epsilon_{t}^{0}(i-1 / 2)^{2},
\end{gathered}
$$

where $\epsilon_{\text {dis }}^{0}=\frac{V_{\text {dis }}^{0}}{2} \exp \left(-\pi^{2} \sqrt{\frac{E_{R}}{V_{0}}}\right), E_{R}=\frac{2 \hbar^{2} \pi^{2}}{m \lambda_{L}^{2}}, \epsilon_{t}^{0}=\frac{\left(\hbar \omega_{t}\right)^{2}}{E_{R}}\left(\frac{\pi}{2}\right)^{2}$.

The on-site interaction parameter can be tuned by varying $a_{s}$, the scattering length of the atoms, using a magnetic field at a Feshbach resonance; or, by varying $\omega_{\perp}$, the transverse trap's angular frequency.

The disorder potential also leads to pseudo-random shifts in the tunneling energy given by:

$$
\Delta J_{i, i+1}=\frac{V_{\text {dis }}^{0}}{2} \exp \left(-\frac{\pi^{2}}{4} \sqrt{\frac{V_{0}}{E_{R}}}\right) \times\left[2+\mathrm{e}^{-\alpha^{2} \sqrt{\frac{E_{R}}{V_{0}}}} \cos (2 \pi \alpha i)+\mathrm{e}^{-\beta^{2} \sqrt{\frac{E_{R}}{V_{0}}}} \cos (2 \pi \beta i)\right] .
$$

Our simulations show that this site-dependence has no influence on the effects we study in the present work; thus, we omit it for simplicity such that $J_{i, i+1} \equiv J$. This is well established in the literature for such models (see e.g., Refs. [17] [40] [41]).

\subsection{The Hartree-Fock-Bogoliubov Formalism}

In this section, we briefly describe a lattice formulation [54] [55] of the finite-temperature HFB [51]-[53] formalism which assumes the existence of BEC. This requirement is fulfilled in the quasi-one-dimensional regime below the critical temperature for finite-size systems as well as in the thermodynamic limit. In the Tonks-Girardeau regime, the global phase coherence is destroyed due to long wavelength fluctuations and the BEC exists only in finite-size systems.

We can therefore assume, in the limit of low temperature, a macroscopic occupation of the ground state such that the Bose annihilation operator can be written as $\hat{a}_{i}=\left(z_{i}+\hat{\delta}_{i}\right) \mathrm{e}^{-i \mu t / \hbar}$, where $z_{i}$ is a complex mean-field part and $\hat{\delta}_{i}$ the fluctuation operator [54]. The condensate density is given by $n_{c, i}=\left|z_{i}\right|^{2}$ and the non-condensate density by $\tilde{n}_{i}=\left\langle\hat{\delta}_{i}^{\dagger} \hat{\delta}_{i}\right\rangle$.

From this, we can derive a discretized, generalized Gross-Pitaevskii equation

$$
\mu z_{i}=\epsilon_{i} z_{i}-J\left(z_{i+1}+z_{i-1}\right)+U\left(n_{c, i}+2 \tilde{n}_{i}\right) z_{i},
$$

coupled to Bogoliubov-de Gennes equations

$$
\hbar \omega_{q} u_{i}^{q}=\left[2 U\left(n_{c, i}+\tilde{n}_{i}\right)-\mu+\epsilon_{i}\right] u_{i}^{q}-J\left[u_{i+1}^{q}+u_{i-1}^{q}\right]-U z_{i}^{2} v_{i}^{q}
$$

and

\footnotetext{
${ }^{1}$ In this approximation, the Wannier functions in the expectation-value integrals are replaced by Gaussians. This gives accurate results for the on-site energies while overestimating the tunneling energy. Since results are given in terms of the tunneling energy $J$, this is of no concern if one keeps in mind that a given value for $J$ does not exactly correspond to the given lattice geometry.
} 


$$
-\hbar \omega_{q} v_{i}^{q}=\left[2 U\left(n_{c, i}+\tilde{n}_{i}\right)-\mu+\epsilon_{i}\right] v_{i}^{q}-J\left[v_{i+1}^{q}+v_{i-1}^{q}\right]-U z_{i}^{2} u_{i}^{q}
$$

through the non-condensate density

$$
\tilde{n}_{i}=\sum_{q}\left[\left|v_{i}^{q}\right|^{2}+\left(\left|u_{i}^{q}\right|^{2}+\left|v_{i}^{q}\right|^{2}\right) N_{B E}\left(\hbar \omega_{q}\right)\right]
$$

where $u_{i}^{q}$ and $v_{i}^{q}$ are the Bogoliubov quasi-particle amplitudes and $N_{B E}$ is the Bose-Einstein distribution. The equations are then solved self-consistently.

We use the phenomenological two-fluid model to define the superfluidity of the condensate [57]. When an external velocity field is applied, via Peierls phase factors on the hopping terms of the Bose-Hubbard Hamiltonian [54] [55], only the inviscid superfluid part will respond; Thus, by comparing the energy of the ground states with and without the external velocity field, we may determine the fraction of atoms that is a superfluid. The superfluid fraction is defined in terms of the condensate and quasi-particle amplitudes as:

$$
f_{s}=f_{s}^{(1)}-f_{s}^{(2)} \text {, }
$$

where

$$
f_{s}^{(1)}=\frac{1}{2 N} \sum_{i}\left[z_{i+1} z_{i}^{*}+z_{i+1}^{*} z_{i}+\sum_{q}\left(v_{i}^{q^{*}} v_{i+1}^{q}+v_{i}^{q} v_{i+1}^{q^{*}}\right)\right]
$$

and

$$
f_{s}^{(2)}=\frac{J}{N} \sum_{q, q^{\prime}}\left(1+\frac{\delta_{q, q^{\prime}}}{2}\right) \frac{\left|\sum_{i}\left(u_{i+1}^{q} v_{i}^{q^{\prime}}-u_{i}^{q} v_{i+1}^{q^{\prime}}\right)\right|^{2}}{\hbar\left(\omega_{q}+\omega_{q^{\prime}}\right)} .
$$

\section{Results}

Here we present results for $500 \mathrm{Rb}-87$ atoms in a system with $N=100$ lattice sites in the experimental configuration of Ref. [16], where $\alpha=55 / 64$ and $\beta=165 / 212$. In what follows, we will use the unit-less parameters $\Delta=\epsilon_{\text {dis }}^{0} / J, V_{t}=\epsilon_{t}^{0} / J$ and $V_{\text {eff }}=U / J$ so that we need not be concerned with evaluating the integrals from experimental parameters.

\subsection{Homogeneous System at Zero Temperature}

To isolate the effects of disorder and interactions, the first simulations are performed without a harmonic trapping potential at $T=0$. We refer to this as a homogeneous system albeit dealing with a finite sized lattice with hard wall boundary conditions.

Figure 1 and Figure 2 show the condensate density for different parameters in linear and logarithmic scale. Panel (a) shows the weakly interacting, non-disordered case, which is just the ground-state of a single particle in a box. Increased disorder then leads to a narrowly peaked density [(b) and (c)]. The logarithmic plot in Figure 2(c) indicates an exponential decay of the condensate density, a signature of Anderson localization. The AndréAubry model [25], which describes non-interacting bosons in two incommensurate lattices (specifically for irrational $\alpha$ ), predicts a transition from extended to localized states for $\Delta=2$. Here we have two secondary lattices with equal amplitudes instead of one and we find that the ground state attains exponentially decaying tails, characteristic of Anderson localization, at $\Delta \approx 1$; However, due to the fact that in our calculations $\alpha$ and $\beta$ are rational and we are dealing with a finite-size system, the transition is broadened [15] [19] and harder to determine with great precision.

In the presence of interactions, the condensate fragments into multiple peaks, each single peak appears to remain exponentially localized (Figure 2(d)); however, other peaks obscure the signature of the tails and the pseudo-period dominates the fine structure. Limited spatial resolution in experiments might also add to the difficulty of characterizing them.

We find that increasing the disorder strength continuously lowers the superfluid fraction, underlining the localizing effect of disorder (Figure 3). The decrease is not dramatic as up to the values of $\Delta$ for those we observe exponential localization (Figure 2(c)) the superfluid fraction remains above 0.85 . 


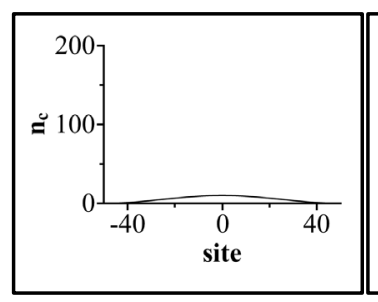

(a)

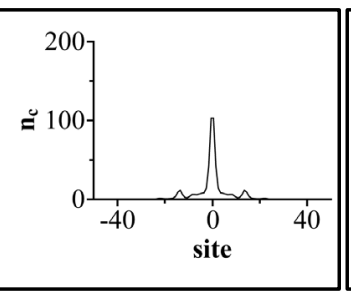

(b)

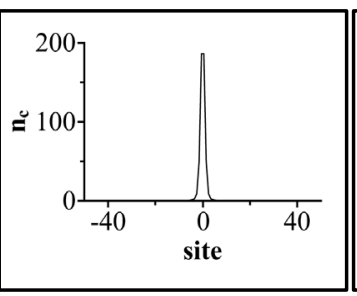

(c)

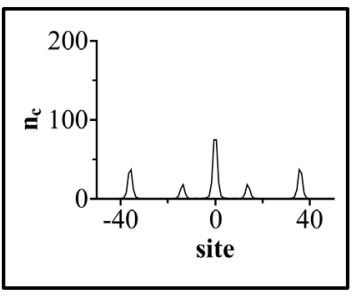

(d)

Figure 1. Condensate density for various disorder and interaction strengths with no harmonic trap at zero temperature. (a) $\Delta=0$; (b) $\Delta=0.68$; (c), (d) $\Delta=1.13$. (a)-(c) $V_{\text {eff }}=10^{-8}$; (d) $V_{\text {eff }}=6 \times 10^{-4}$.

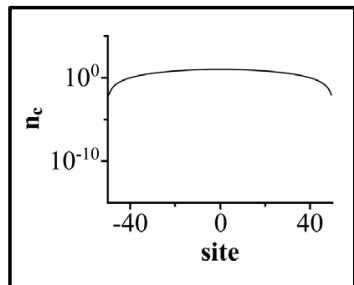

(a)

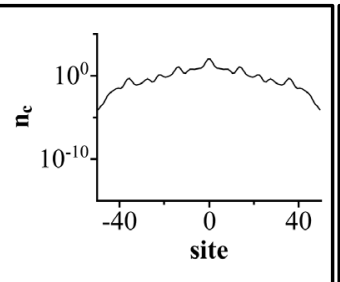

(b)

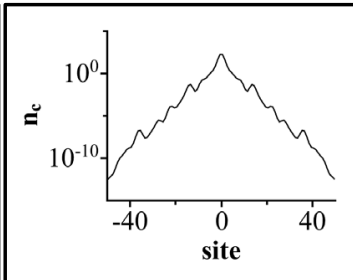

(c)

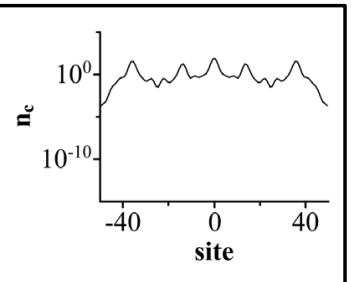

(d)

Figure 2. Condensate density (logarithmic) for various disorder and interaction strengths with no harmonic trap at zero temperature. (a) $\Delta=0$; (b) $\Delta=0.68$; (c), (d) $\Delta=1.13$. (a)-(c) $V_{\text {eff }}=10^{-8}$; (d) $V_{\text {eff }}=6 \times 10^{-4}$.

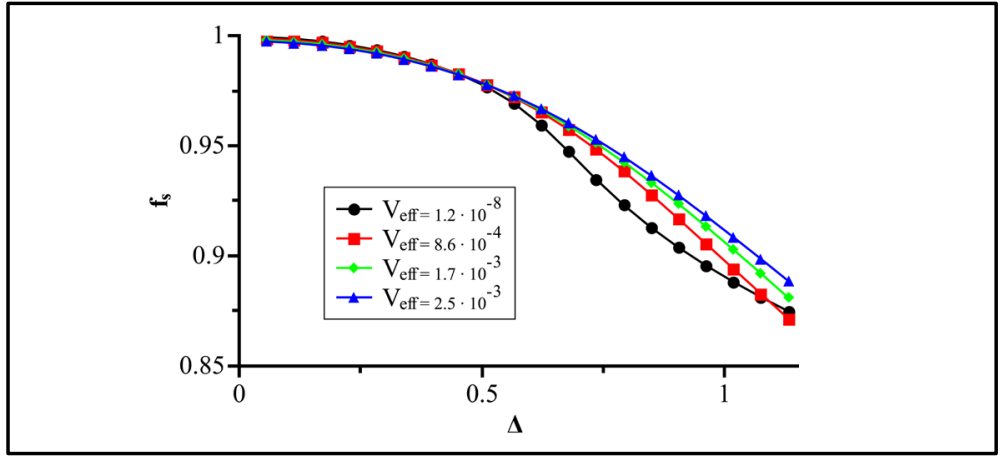

Figure 3. (Colour online) Superfluid fraction vs. disorder strength at various interaction strengths with no harmonic trap at zero temperature.

In the case of weak disorder, the superfluid fraction decreases slightly with increased interaction strength, which is explained by repulsive interactions inhibiting superfluidity. In contrast, for increased disorder the superfluid fraction increases with higher $V_{\text {eff }}$. This follows from the interplay between interactions and disorder. The disorder localizes the condensate, thereby reducing the superfluid fraction. The interactions delocalize the condensate and thus attenuate this effect. We note that for very large interaction strengths, the superfluid fraction will decrease again [58], which is not described by the HFB formalism.

Both the superfluid fraction and the lowest excitation energy show a sharp drop in the region where the interactions begin to fragment the condensate (Figure 4). The lowest excitation energy approaches zero where the energies for the single-peaked state and the fragmented state are equal. This effect results from the competition between the on-site energy and the kinetic and interaction energies. The single large peak minimizes the external potential energy while a fragmented condensate minimizes the kinetic and interaction energy. Above a critical interaction strength, the increased potential energy is compensated by the reduced kinetic and interaction energies. As each peak is exponentially localized, the superfluid fraction decreases at the critical interaction strength, but afterwards continues to increase as the interactions keep delocalizing the condensate.

The excitation spectrum is shown in Figure 5 for a weakly interacting condensate with and without disorder. In the absence of disorder, we obtain the energy spectrum of a free particle in a sinusoidal potential. In the pres- 


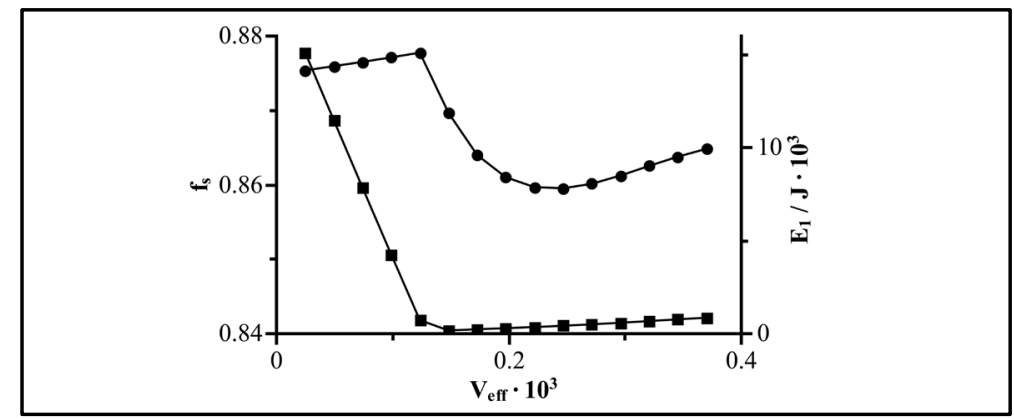

Figure 4. Superfluid fraction ( $f_{s}$ : circles) and lowest excitation energy ( $E_{1}$ : squares) vs. interaction strength with disorder $(\Delta=1.13)$ and no harmonic trap at zero temperature.

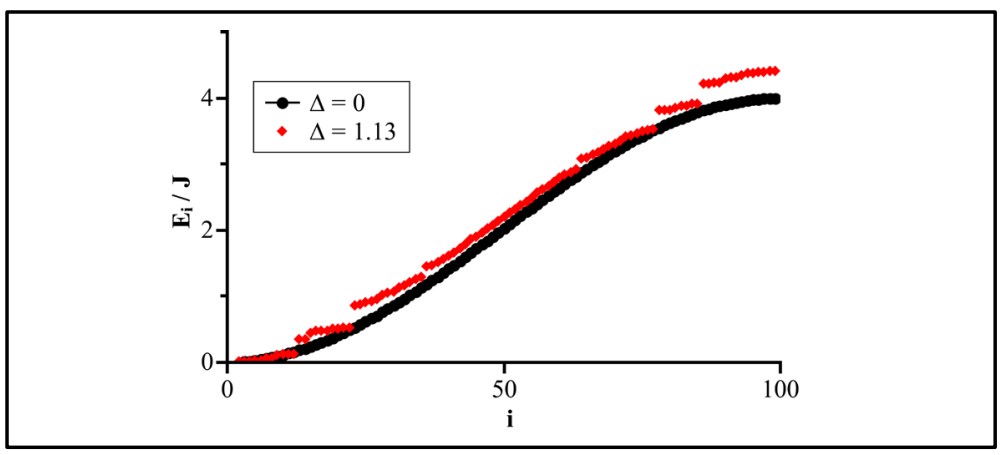

Figure 5. (Colour online) Energy spectrum of the weakly interacting con= densate $\left(V_{\text {eff }}=10^{-8}\right)$ with and without disorder and absent harmonic trap at zero temperature.

ence of disorder, the spectrum shows gaps at fractions corresponding to the lattice ratios $\alpha$ and $\beta$, demonstrating the quasi-periodicity of disorder. In the setup of [16], for example, $\alpha$ is approximately 0.86 and $\beta$ is approximately 0.78 . We find gaps, for example, at indices 86 and $100-86_{1}=14$ due to $\alpha$, at 78 and $100-78=22$ due to $\beta$ and at 35 due to a beating, where $0.35=2\left[\alpha^{-1}+(1-\beta)^{-1}\right]^{-1}$. Our simulations show that $V_{\text {eff }}$ has no qualitative effect on the energy spectrum. We conclude that the excitation spectrum is primarily governed by single-particle effects.

\subsection{Harmonic Trap at Zero Temperature}

The general observations of the previous section remain valid when a harmonic trapping potential is present, with the main difference being the additional confinement due to the trap. We choose a trap strength, $V_{t}$, equivalent to $f_{t}=10 \mathrm{~Hz}$ in the Schulte setup [16]. In this case, only in the logarithmic plots is it possible to distinguish between localization due to the trap, which is Gaussian, and Anderson localization, which is exponential (Figure 6). We also note that the trap inhibits the fragmentation of the condensate. This can be seen most clearly in the graphs for the superfluid fraction and the lowest excitation energy (Figure 7). In contrast to the homogeneous case, no sharp drops occur and the lowest excitation energy does not approach zero. We conclude that no sudden crossing from a single-peak state to a multiple-peak state occurs. Instead, side peaks arise continuously over a wide range of interaction strengths.

\subsection{Harmonic Trap at Finite Temperature}

We now discuss the situation for finite temperature. For the mean-field approximation to remain valid, we must stay well below the critical temperature where the non-condensate density is low. For our simulations, we take the setup from the previous section and vary the temperature such that $k_{B} T / J=8.89$ (equivalent to $10 \mathrm{nK}$ in the Schulte [16] setup). 


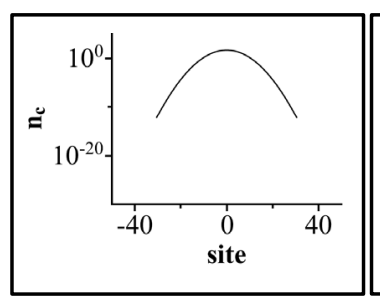

(a)

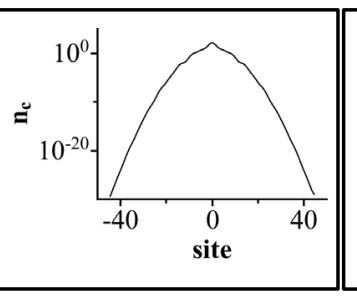

(b)

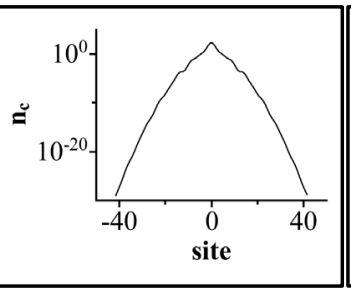

(c)

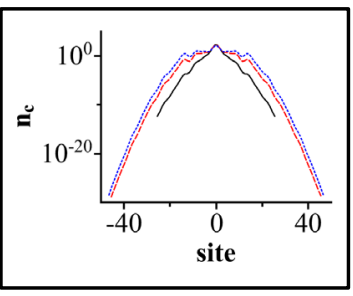

(d)

Figure 6. (Colour online) Condensate density (logarithmic) in a harmonic trap $\left(V_{t}=3.22 \times 10^{-3}\right)$ for various disorder and interaction strengths at zero temperature. (solid black) $V_{\text {eff }}=10^{-8}$. (dashed red) $V_{\text {eff }}=3.7 \times 10^{-4}$. (dotted blue) $V_{\text {eff }}=6.2 \times 10^{-4}$. (a) $\Delta=0$; (b) $\Delta=0.68$; (c), (d) $\Delta=1.13$.

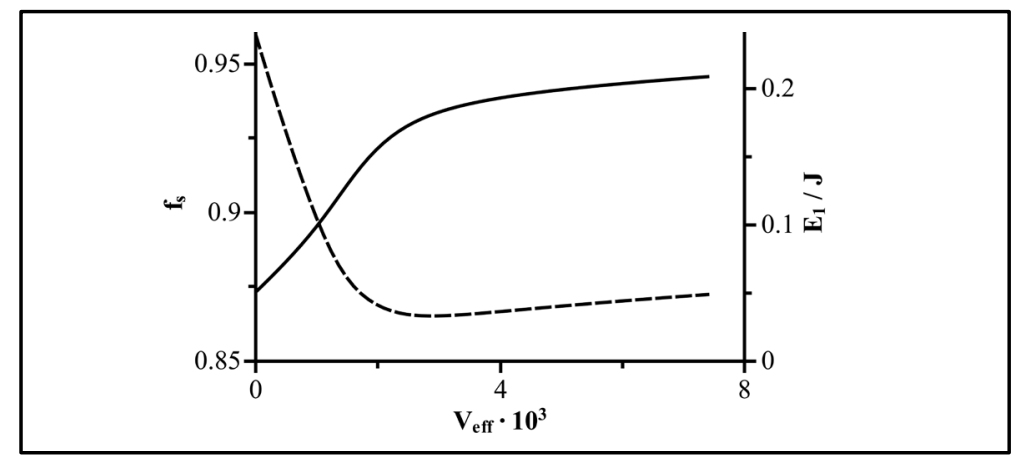

Figure 7. Superfluid fraction $\left(f_{s}\right.$ : solid line) and lowest excitation energy $\left(E_{1}\right.$ dashed line) vs. interaction strength with no disorder in a harmonic trap at zero temperature.

The effect of the finite temperature is to drive atoms out of the condensate into the non-condensate. Thus the condensate fraction $f_{c}$ is lowered and the non-condensate density $\tilde{n}$ is increased. Because Anderson localization is a single-particle effect that does not depend on the total number of atoms in the condensate, we still see a Gaussian peak for weak disorder and exponential localization for strong disorder. The non-condensate, while being slightly affected by the disorder potential, does not become localized (Figure 8). This is because the noncondensate is incoherent and therefore behaves more like a classical gas.

The interplay between disorder and interactions is more subtle at finite temperature. We first observe that increasing the interaction strength does not fragment the condensate. Instead, atoms are driven into the non-condensate (Figure 9). This is energetically advantageous as the non-condensate has lower interaction energy due to its reduced density. We further see that disorder increases the condensate fraction for weak interactions and decreases it for strong interactions. The former is a consequence of the disorder lowering the density of states at low energy (Figure 5) thus stabilizing condensation at finite temperature. For strong interactions, in contrast, the localizing effect of disorder increases the interaction energy, thereby driving more atoms into the non-condensate.

We conclude the treatment of the harmonically confined Bose gas at finite temperature with a discussion of the excitation energies. Again, we look at the interplay of temperature, disorder and interactions.

First, we investigate the temperature dependence. Our simulations show that for weak interactions $\left(V_{\text {eff }} \sim 10^{-8}\right)$, the temperature has essentially no effect on the band structure, regardless of the value of $\Delta$. This is not surprising as temperature does not alter the energy levels or states of a single particle system. In the presence of interactions, however, we see that increasing the temperature also increases the lowest excitation energy (Figure 10), and this effect becomes more pronounced as $V_{\text {eff }}$ increases. This can be explained with the simultaneous decrease in the condensate fraction as temperature and interaction strength increase. Removing atoms from the condensate lowers the energy of the ground state via the interaction contribution $n_{c}^{2} V_{\text {eff }}$ and raises the energy of the excited states, thereby increasing the energy difference $E_{1}$ between the ground state and the first excited state. 


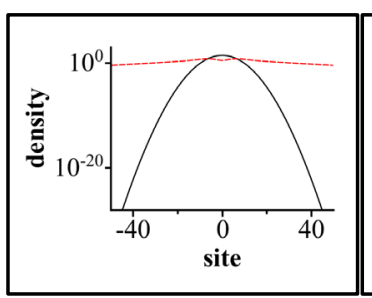

(a)

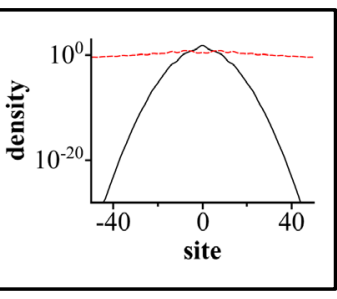

(b)

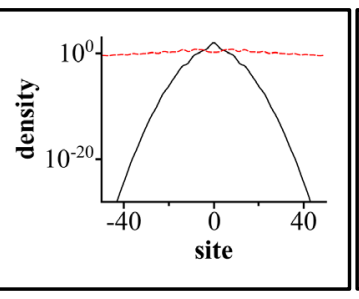

(c)

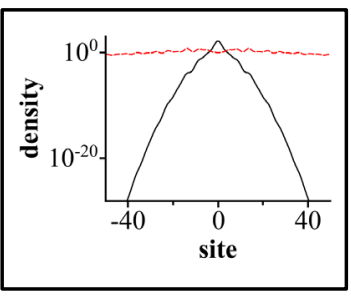

(d)

Figure 8. (Colour online) Logarithmically scaled condensate density (solid black) and non-condensate density (dashed red) in a harmonic trap $\left(V_{t}=3.22 \times 10^{-3}\right)$ for various disorder strengths at $k_{B} T / J=8.89$ and weak interactions $\left(V_{\text {eff }}=10^{-8}\right)$. (a) $\Delta=0$; (b) $\Delta=0.45$; (c) $\Delta=0.68$; (d) $\Delta=1.13$.

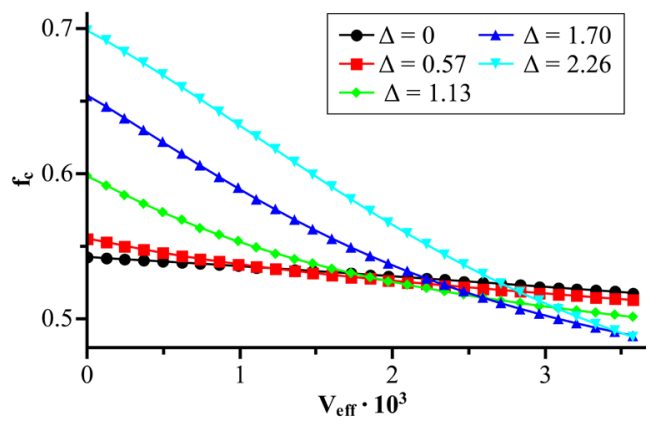

Figure 9. (Colour online) Condensate fraction vs. interaction strength at $k_{B} T / J=8.89$ for various disorder strengths in a harmonic trap.

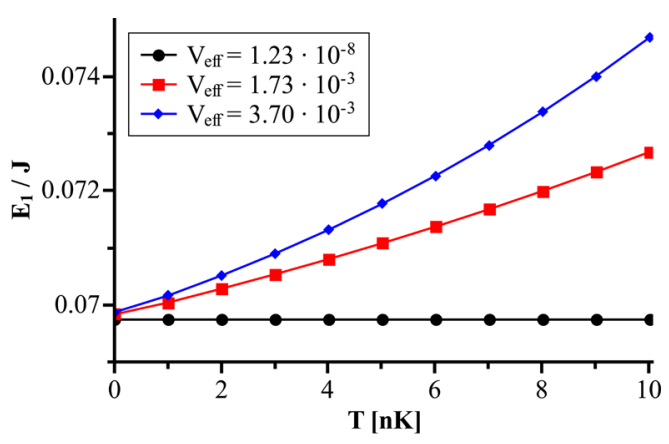

Figure 10. (Colour online) Lowest excitation energy vs. temperature for various interaction strengths with no disorder potential in a harmonic trap.

Next, we turn to the interplay between disorder and interactions. We expect that at constant temperature, any change that increases $E_{1}$ will also increase $f_{c}$. This is because more thermal energy is required to populate the low lying levels. Comparing Figure 9 and Figure 11, this trend seems to hold for varying disorder, however, increasing $V_{\text {eff }}$ tends to increase $E_{1}$ while decreasing $f_{c}$. This is attributed to the non-linear density dependence of the interaction energy as discussed above.

The effect of disorder in the weakly interacting regime is to increase the excitation energy. For higher interaction strengths, this effect is reversed reflecting the fact that more atoms leave the condensate when it becomes localized in the presence of strong interactions.

\subsection{Summary}

In summary, in Subsection 3.1 we studied the ultracold disordered Bose gas in a 1D lattice with hard wall boun- 


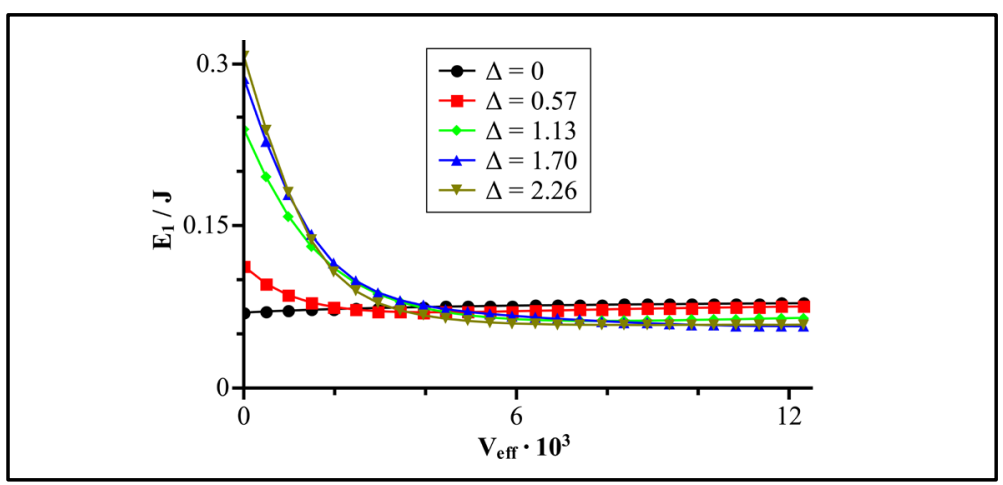

Figure 11. (Colour online) Lowest excitation energy vs. interaction strength for various disorder strengths at $k_{B} T / J=8.89$.

dary conditions. We noted that, in the absence of interactions, the André-Aubry model pertains. The main effects of interactions over and above those of the disorder were to fragment the condensate as interactions were increased and also to reduce the level of localization which could be seen through an increase in the superfluid fraction.

In Section 3.2, these results were extended to the case with a harmonic trap at zero temperature. It was noted that it remained possible to distinguish between the Gaussian profile due to harmonic confinement and the Lorentzian character of the localized states. The sharp fragmentation with increasing interactions observed in 3.1 was not present in the harmonic trap however, and we argue that this is due to the breaking of the translational symmetry which leads to multiple degeneracies in the ground state in 3.1.

Finally, in 3.3 we investigated the effects of temperature upon the harmonically trapped gas. Qualitatively, the effects of disorder (and interactions) upon the condensate were seen to be similar to the case at zero temperature, however an interesting interplay between interactions and disorder upon the condensate fraction was observed.

\section{Conclusion}

In this paper, we have discussed the interacting ultra-cold Bose gas in incommensurate optical lattices and highlighted the important role of disorder, interactions, harmonic trapping, and temperature in the quest for Anderson localization. At zero temperature and in the absence of the harmonic trap, we find a transition from extended to exponentially localized states for weak interactions. For stronger interactions, the condensate becomes fragmented and exponentially localized fragments ovelap. The superfluid fraction decreases with the interaction in the case of weak disorder but increases if the disorder is strong. The presence of the harmonic trap inhibits the condensate fragmentation at zero temperature. The main effect of the temperature is to drive atoms into the noncondensate which is slightly affected by the disorder and does not become localized. The increase of the interaction at finite temperature does not fragment the condensate but leads to the growth of the delocalized non-condensate.

\section{Acknowledgements}

This work was supported by contract SFB/TR 12 of the German Research Foundation and through the IB BMBF (Project NZL 07/006) and by the New Zealand Foundation for Research, Science and Technology through contract NERF-UOOX0703: Quantum Technologies and the New Zealand International Science and Technology Linkages Fund. JT and DAWH also acknowledge support from the National Research Foundation and Ministry of Education of Singapor.

\section{References}

[1] Morsch, O. and Oberthaler, M. (2006) Reviews of Modern Physics, 78, 179-215. http://dx.doi.org/10.1103/RevModPhys.78.179

[2] Lewenstein, M., Sanpera, A., Ahufinger, V., Damski, B., Sen(De), A. and Sen, U. (2007) Advances in Physics, 56, 243-379. http://dx.doi.org/10.1080/00018730701223200 
[3] Fallani, L., Fort, C. and Inguscio, M. (2008) Advances in Atomic, Molecular, and Optical Physics, 56, 119-160. http://dx.doi.org/10.1016/S1049-250X(08)00012-8

[4] Bloch, I., Dalibard, J. and Zwerger, W. (2008) Reviews of Modern Physics, 80, 885-964. http://dx.doi.org/10.1103/RevModPhys.80.885

[5] Sanchez-Palencia, L. and Lewenstein, M. (2010) Nature Physics, 6, 87-95. http://dx.doi.org/10.1038/nphys1507

[6] Modugno, G. (2010) Reports on Progress in Physics 73, Article ID: 102401. http://dx.doi.org/10.1088/0034-4885/73/10/102401

[7] Inouye, S., Andrews, M.R., Stenger, J., Miesner, H.-J., Stamper-Kurn, D.M. and Ketterle, W. (1998) Nature (London), 392, 151-154. http://dx.doi.org/10.1038/32354

[8] Olshanii, M. (1998) Physical Review Letters, 81, 938-941. http://dx.doi.org/10.1103/PhysRevLett.81.938

[9] Dunjko, V., Lorent, V. and Olshanii, M. (2001) Physical Review Letters, 86, 5413-5416. http://dx.doi.org/10.1103/PhysRevLett.86.5413

[10] Haller, E., Gustavsson, M., Mark, M.J., Danzl, J.G., Hart, R., Pupillo, G. and Näger, H.-C. (2009) Science, 325, 1224-1227. http://dx.doi.org/10.1126/science.1175850

[11] Schulte, T., Drenkelforth, S., Kruse, J., Ertmer, W., Arlt, J., Sacha, K., Zakrzewski, J. and Lewenstein, M. (2005) Physical Review Letters, 95, Article ID: 170411. http://dx.doi.org/10.1103/PhysRevLett.95.170411

[12] White, M., Pasienski, M., McKay, D., Zhou, S.Q., Ceperley, D. and DeMarco, B. (2009) Physical Review Letters, 102, Article ID: 055301. http://dx.doi.org/10.1103/PhysRevLett.102.055301

[13] Pasienski, M., McKay, D., White, M. and DeMarco, B. (2010) Nature Physics, 6, 677-680. http://dx.doi.org/10.1038/nphys1726

[14] Fallani, L., Lye, J.E., Guarrera, V., Fort, C. and Inguscio, M. (2007) Physical Review Letters, 98, Article ID: 130404. http://dx.doi.org/10.1103/PhysRevLett.98.130404

[15] Roati, G., D’Errico, C., Fallani, L., Fattori, M., Fort, C., Zaccanti, M., Modugno, G., Modugno, M. and Inguscio, M. (2008) Nature (London), 453, 895-898. http://dx.doi.org/10.1038/nature07071

[16] Schulte, T., Drenkelforth, S., Kruse, J., Tiemeyer, R., Sacha, K., Zakrzewski, J., Lewenstein, M., Ertmer, W. and Arlt, J.J. (2006) New Journal of Physics, 8, 230. http://dx.doi.org/10.1088/1367-2630/8/10/230

[17] Guarrera, V., Fallani, L., Lye, J.E., Fort, C. and Inguscio, M. (2007) New Journal of Physics, 9, 107. http://dx.doi.org/10.1088/1367-2630/9/4/107

[18] Guidoni, L., Triché, C., Verkerk, P. and Grynberg, G. (1997) Physical Review Letters, 79, 3363-3366. http://dx.doi.org/10.1103/PhysRevLett.79.3363

[19] Lye, J.E., Fallani, L., Fort, C., Guarrera, V., Modugno, M., Wiersma, D.S. and Inguscio, M. (2007) Physical Review A, 75, Article ID: 061603(R). http://dx.doi.org/10.1103/PhysRevA.75.061603

[20] Deissler, B., Zaccanti, M., Roati, G., D’Errico, C., Fattori, M., Modugno, M., Modugno, G. and Inguscio, M. (2010) Nature Physics, 6, 354-358. http://dx.doi.org/10.1038/nphys1635

[21] Deissler, B., Lucioni, E., Modugno, M., Roati, G., Tanzi, L., Zaccanti, M., Inguscio, M. and Modugno, G. (2011) New Journal of Physics, 13, Article ID: 023020. http://dx.doi.org/10.1088/1367-2630/13/2/023020

[22] Lucioni, E., Deissler, B., Tanzi, L., Roati, G., Zaccanti, M., Modugno, M., Larcher, M., Dalfovo, F., Inguscio, M. and Modugno, G. (2011) Physical Review Letters, 106, Article ID: 230403. http://dx.doi.org/10.1103/PhysRevLett.106.230403

[23] Gadway, B., Pertot, D., Reeves, J., Vogt, M. and Schneble, D. (2011) Physical Review Letters, 107, Article ID: 145306. http://dx.doi.org/10.1103/PhysRevLett.107.145306

[24] Anderson, P.W. (1958) Absence of Diffusion in Certain Random Lattices. Physical Review, 109, 1492-1505. (For a review, see Kramer, B. and MacKinnon, A. (1993) Localization: Theory and experiment. Reports on Progress in Physics, 56, 1469.) http://dx.doi.org/10.1088/0034-4885/56/12/001

[25] Aubry, S. and André, G. (1980) Annals of the Israel Physical Society, 3, 133.

[26] Harper, P.G. (1955) Proceedings of the Physical Society. Section A, 68, 874. http://dx.doi.org/10.1088/0370-1298/68/10/304

[27] Grempel, D.R., Fishman, S. and Prange, R.E. (1982) Physical Review Letters, 49, 833-836. http://dx.doi.org/10.1103/PhysRevLett.49.833

[28] Kohmoto, M. (1983) Physical Review Letters, 51, 1198-1201. http://dx.doi.org/10.1103/PhysRevLett.51.1198

[29] Diener, R.B., Georgakis, G.A., Zhong, J., Raizen, M. and Niu, Q. (2001) Physical Review A, 64, Article ID: 033416. 
http://dx.doi.org/10.1103/PhysRevA.64.033416

[30] Ingold, G.L., Wobst, A., Aulbach, Ch. and Hänggi, P. (2002) European Physical Journal B, 30, 175-179. http://dx.doi.org/10.1140/epjb/e2002-00372-9

[31] Boers, D.J., Goedeke, B., Hinrichs, D. and Holthaus, M. (2007) Physical Review A, 75, Article ID: 063404. http://dx.doi.org/10.1103/PhysRevA.75.063404

[32] Biddle, J. and Sarma, S.D. (2010) Physical Review Letters, 104, Article ID: 070601. http://dx.doi.org/10.1103/PhysRevLett.104.070601

[33] Larcher, M., Modugno, M. and Dalfovo, F. (2011) Physical Review A, 83, Article ID: 013624. http://dx.doi.org/10.1103/PhysRevA.83.013624

[34] Larcher, M., Dalfovo, F. and Modugno, M. (2009) Physical Review A, 80, Article ID: 053606. http://dx.doi.org/10.1103/PhysRevA.80.053606

[35] Zhang, Z., Tong, P., Gong, J. and Li, B. (2011) Physical Review E, 83, Article ID: 056205. http://dx.doi.org/10.1103/PhysRevE.83.056205

[36] Adhikari, S.K. and Salasnich, L. (2009) Physical Review A, 80, Article ID: 023606. http://dx.doi.org/10.1103/PhysRevA.80.023606

[37] Sanchez-Palencia, L. and Santos, L. (2005) Physical Review A, 72, Article ID: 053607. http://dx.doi.org/10.1103/PhysRevA.72.053607

[38] Cetoli, A. and Lundh, E. (2010) Physical Review A, 81, Article ID: 063635. http://dx.doi.org/10.1103/PhysRevA.81.063635

[39] Shrestha, U. and Modugno, M. (2010) Physical Review A, 82, Article ID: 033604. http://dx.doi.org/10.1103/PhysRevA.82.033604

[40] Roscilde, T. (2008) Physical Review A, 77, Article ID: 063605. http://dx.doi.org/10.1103/PhysRevA.77.063605

[41] Schmitt, F., Hild, M. and Roth, R. (2009) Physical Review A, 80, Article ID: 023621. http://dx.doi.org/10.1103/PhysRevA.80.023621

[42] Deng, X., Citro, R., Minguzzi, A. and Orignac, E. (2008) Physical Review A, 78, Article ID: 013625. http://dx.doi.org/10.1103/PhysRevA.78.013625

[43] Roux, G., Barthel, T., McCulloch, I.P., Kollath, C., Schollwöck, U. and Giamarchi, T. (2008) Physical Review A, 78, Article ID: 023628. http://dx.doi.org/10.1103/PhysRevA.78.023628

[44] Deng, X., Citro, R., Orignac, E. and Minguzzi, A. (2009) European Physical Journal B, 68, 435-443. http://dx.doi.org/10.1140/epjb/e2009-00069-7

[45] Rey, A.M., Satija, I.I. and Clark, C.W. (2006) Physical Review A, 73, Article ID: 063610. http://dx.doi.org/10.1103/PhysRevA.73.063610

[46] Cai, X., Chen, S. and Wang, Y. (2010) Superfluid-to-Bose-glass transition of hard-core bosons in a one-dimensional incommensurate optical lattice. Physical Review A, 81, Article ID: 023626.

[47] Cai, X., Chen, S. and Wang, Y. (2010) Ground-state and dynamical properties of hard-core bosons in one-dimensional incommensurate optical lattices with a harmonic trap. Physical Review A, 81, Article ID: 053629.

[48] Towers, J., Cormack, S.C. and Hutchinson, D.A.W. (2013) Physical Review A, 88, Article ID: 043625. http://dx.doi.org/10.1103/PhysRevA.88.043625

[49] Jachymski, K. and Idziaszek, Z. (2012) Physical Review A, 86, Article ID: 023607. http://dx.doi.org/10.1103/PhysRevA.86.023607

[50] Pethick, C.J. and Smith, H. (2002) Bose-Einstein Condensation in Dilute Gases. Cambridge University Press, Cambridge.

[51] Griffin, A. (1996) Physical Review B, 53, 9341-9347. http://dx.doi.org/10.1103/PhysRevB.53.9341

[52] Hutchinson, D.A.W., Zaremba, E. and Griffin, A. (1997) Physical Review Letters, 78, 1842-1845. http://dx.doi.org/10.1103/PhysRevLett.78.1842

[53] Hutchinson, D.A.W., Burnett, K., Dodd, R.J., Morgan, S.A., Rusch, M., Zaremba, E., Proukakis, N.P., Edwards, M. and Clark, C.W. (2000) Journal of Physics B, 33, 3825. http://dx.doi.org/10.1088/0953-4075/33/19/302

[54] Wild, B.G., Blakie, P.B. and Hutchinson, D.A.W. (2006) Physical Review A, 73, Article ID: 023604. http://dx.doi.org/10.1103/PhysRevA.73.023604

[55] Rey, A.M., Burnett, K., Roth, R., Edwards, M., Williams, C.J. and Clark, C.W. (2003) Journal of Physics B, $36,825$. http://dx.doi.org/10.1088/0953-4075/36/5/304 
[56] van Oosten, D., van der Straten, P. and Stoof, H.T.C. (2001) Physical Review A, 63, Article ID: 053601. http://dx.doi.org/10.1103/PhysRevA.63.053601

[57] Lieb, E.H., Seiringer, R. and Yngvason, J. (2002) Physical Review B, 66, Article ID: 134529. http://dx.doi.org/10.1103/PhysRevB.66.134529

[58] Batrouni, G.G. and Scallettar, R.T. (1992) Physical Review B, 46, 9051-9062. http://dx.doi.org/10.1103/PhysRevB.46.9051 